\title{
Semantic activation in LSD: evidence from picture naming
}

Neiloufar Family ${ }^{1}$, David Vinson ${ }^{2}$, Gabriella Vigliocco ${ }^{2}$, Mendel Kaelen ${ }^{3}$,

Mark Bolstridge ${ }^{3}$, David J Nutt ${ }^{3}$, Robin L Carhart-Harris ${ }^{3}$

${ }^{I}$ Department of Psycholinguistics and Language Development, University of Kaiserslautern, Kaiserslautern, Germany

${ }^{2}$ Department of Experimental Psychology, University College London, London, United Kingdom

${ }^{3}$ Centre for Neuropsychopharmacology, Imperial College London, London, United Kigndom

Neiloufar Family (corresponding author)

family@rhrk.uni-kl.de

Psycholinguistics and Language Development

Faculty of Social Sciences

University of Kaiserslautern

Erwin-Schrödinger-Straße, Bldg. 57

67663 Kaiserslautern

Germany

+496312054138 


\section{Semantic activation in LSD: evidence from picture naming}

LSD is a classic psychedelic drug that alters cognition in a characteristic way. It has been suggested that psychedelics expand the breadth of cognition via actions on the central nervous system. Previous work has shown changes in semantic processing under psilocybin (a related psychedelic to LSD) that are consistent with an increased spread of semantic activation. The present study investigates this further using a picture naming task and the psychedelic, LSD. Ten participants completed the task under placebo and LSD. Results revealed significant effects of LSD on accuracy and error correction that were consistent with an increased spread of semantic activation under LSD. These results are consistent with a generalized "entropic" effect on the mind. We suggest incorporating direct neuroimaging measures in future studies, and to employ more naturalistic measures of semantic processing that may enhance ecological validity.

Keywords: psychedelics, lysergic acid diethylamide, speech errors, production, psychopharmacology

\section{Introduction}

This study aimed to explore the effects of lysergic acid diethylamide, a serotonergic hallucinogen, on semantic processing and more specifically, lexical (i.e., word) retrieval. Previous drug studies have shown that modulation of neurotransmitter systems have upstream effects on language processing (Aarsland, Larsen, Reinvang, \& Aasland, 1994; Kischka et al., 1996; Rosenberger, 1980). Combining picture naming or priming paradigms with activation of particular neuronal pathways allows us to explore the role of particular neurotransmitters in modulating lexical retrieval, which can be informative about current theories of language production.

\section{Lysergic Acid Diethylamide}

Research on LSD and related serotonergic "psychedelics" can provide novel 
perspectives on the relationship between human brain activity and cognition (CarhartHarris et al., 2014a). Studies to-date have focused on mechanisms of action (GouzoulisMayfrank et al., 1998a; Carhart-Harris et al., 2014b), pharmacokinetics (Hasler et al., 2004), therapeutics (Gasser et al., 2014), and their ability to model psychosis (Geyer \& Vollenweider, 2008). Psychedelic drugs possess a similar molecular structure to the endogenous neurotransmitter serotonin (5-HT) and trigger characteristic changes in perception and cognition via activation of the $5-\mathrm{HT}_{2 \mathrm{~A}}$ receptor (Halberstadt \& Geyer, 2011). Evidence suggests an involvement of the 5- $\mathrm{HT}_{2 \mathrm{~A}}$ receptor in schizophrenia (Raote, Bhattacharya, \& Panicker, 2007), a mental disorder partly characterized by impairments in language production (Sundheim \& Voeller, 2004). 5-HT pathways have also been found in language areas via PET imaging (Fink et al., 2009). Animal and human research has shown that the 5- $\mathrm{HT}_{2 \mathrm{~A}}$ receptor in particular is critically involved in associative learning (Harvey, 2003), working memory (Williams et al., 2002), visual processing, and emotion regulation (Kometer et al., 2011). These findings motivate using LSD as a tool to investigate the mechanics of linguistic processing.

\section{Neurochemistry and Language}

While studies on the neurobiology of language have mostly focused on impairment in patient populations (Albert, 2000), a small number of studies have looked at pharmacological modulation of semantic network activation. For example, Kischka et al. (1996) showed that the dopamine agonist $l$-dopa reduces indirect semantic priming in healthy humans, marked by an increase in reaction times to indirectly related word pairs (e.g. stimuli pairs that have intervening semantic nodes like tiger for the pair lionstripes) in a lexical decision task. Copland et al. (2003) also showed that l-dopa decreases semantic priming for pairs of words where one of the words has either a dominant or subordinate homonym meaning. Both these studies suggest that dopamine 
plays a regulatory role in semantic activation, enhancing 'focus' and restricting access to a diffuse semantic network.

In contrast, Spitzer et al. (1996) demonstrated that the mixed 5-HT receptor agonist psilocybin, a closely related molecule to LSD, enhances indirect semantic priming. Subjects showed facilitation in responses to a verbal stimulus following an indirectly related prime. These findings were in line with reports from the 60 s that showed that serotonergic hallucinogens (e.g. LSD, psilocybin, mescaline) render speech less predictable and enhance free-association (e.g. Amarel \& Cheek, 1965; Landon \& Fischer, 1970). This is also consistent with a recent hypothesis on the action of psychedelics on the mind and brain, informed by modern neuroimaging studies, known as the "entropic brain" hypothesis (Carhart-Harris et al., 2014a). In brief, this hypothesis states that brain activity and associated psychological functions becomes less predictable in the psychedelic state.

Spitzer et al. (1996) argued that the semantic effects of psilocybin were timesensitive, only appearing 50 minutes after ingestion, closely resembling the temporal profile of plasma levels of psilocin (i.e. the active metabolite of psilocybin) as shown in Figure 1. (Figure 1 goes here)

However, these results are unclear, as the authors do not report tests of the crucial interaction (drug vs. placebo $\times$ time) for the most appropriate comparison with direct semantic priming. Moreover, if the levels of plasma psilocin predict indirect semantic priming, it should have also been observable 150 minutes post-ingestion when psilocin levels remained high. In fact, a later study (Gouzoulis-Mayfrank et al., 1998b) attempted to replicate these findings and only found a trend for increased indirect semantic priming. Thus, taken together, these studies preclude us from drawing any firm conclusions about psychedelic's effects on indirect semantic priming, while 
suggesting that they do serve to enhance it, consistent with a generic defocusing, hyperassociative effect.

\section{Current Study}

In the present study, the effects of LSD and placebo were compared in a picture naming task with the aim of assessing Spitzer et al.'s (1996) proposal that psychedelic drugs serve to modulate the activation of semantic networks. LSD has a similar mechanism of action (i.e. high affinity to the 5- $\mathrm{HT}_{2 \mathrm{~A}}$ receptor), and similar subjective and behavioural effects to psilocybin (Wolbach, Miner, \& Isbell, 1962). However, LSD has a longer half-life (Aghajanian \& Bing, 1964), which allows for flexible timing in testing, as the plasma levels of LSD decrease at a slower rate. Plasma levels and correlated behavioural effects can still be detected at 8 hours post-dose (Aghajanian \& Bing, 1964). Furthermore, picture naming paradigms offer us a richer data set of errors produced alongside reaction times: errors can reveal details of the cognitive processes involved in lexical retrieval that are affected by a particular manipulation (Fromkin, 1971). Importantly, semantically-related lexical substitution errors (e.g. saying "cat" when "dog" is intended) may reflect spread of semantic activation around a target concept (e.g. Garrett, 1992).

In language production, naming a picture takes longer and is more prone to errors when named in the context of other pictures from the same category than when in the context of pictures from different categories (Damian, Vigliocco, \& Levelt, 2001; Kroll \& Stewart, 1994). This semantic context effect is not discrete but graded: when a picture is named in the context of near category pictures (e.g. body parts and articles of clothing that are relatively similar categories), interference is reduced (Vigliocco et al., 2002). The difference in reaction times in semantically same conditions vs. far conditions has been shown in different production tasks (e.g. Schriefers, Meyer, \& 
Levelt, 1990). Under semantic competition accounts of lexical retrieval in production, words compete to be selected on the basis of their meanings, and words that are more closely related, compete more. Words that are further apart in the semantic network would be less likely to compete as a function of their distance (Damian et al., 2001). Other accounts have attributed this effect to incremental learning and repetition priming (see Navarrete, Mahon, \& Caramazza, 2010).

We hypothesised that LSD would have a similar effect to psilocybin on semantic network activation: slowing and increasing error rates in picture naming, as well as the pattern of errors described above. Under placebo (baseline), we expected to replicate the context effects of Vigliocco et al. (2002), i.e. reaction times would be faster for far category items, intermediate for near category items, and slowest for same category items. If LSD increases semantic network activation, we expect an increase in reaction times, and especially so for the near category items if activation spreads more broadly than just within a semantic category, rendering them more similar to the same category items. These results would be in line with findings from Spitzer et al. (1996) for psilocybin. Furthermore, we would predict both more errors from the same category due to stronger activation and competition in nearby lexical items which are already plausible competitors, but also more errors from outside the target category if activation spreads sufficiently broadly to more distant items.

\section{Method}

\section{Participants}

Ten healthy volunteers (males $=9$; mean age $=34.2 \pm 7.4$, range $=26-47$; nativespeakers of English $=8$ ) participated in this pilot study. Travel expenses to the testing 
site were reimbursed but there was no other financial incentive. All subjects had used at least one psychedelic drug in their lives and all but one had used LSD (mean lifetime uses of $\mathrm{LSD}=65 \pm 90$, range $=0-250$ ). None of the subjects had ingested any psychoactive drugs in the 6 weeks prior to the experiment sessions. Participants underwent a screening prior to the first test session, which included a psychiatric interview, routine blood analysis, urine analysis, electrocardiogram, blood pressure and heart rate, and a neuropsychological examination. After being briefed in writing and orally of the aims and procedures of the study, participants were required to provide written consent in order to participate.

This study was approved by the NRES committee London-West London and was conducted in accordance with the revised declaration of Helsinki, the International Committee on Harmonisation Good Clinical Practice guidelines and NHS Research Governance Framework. Imperial College London sponsored the research and a Home Office license was obtained for research with schedule one drugs.

\section{Materials}

The materials used in this experiment were identical to those used in the object naming experiment in Vigliocco et al. (2002), with most of the 24 pictures coming from Snodgrass and Vanderwart (1980). In order to test graded semantic effects, the images of objects included three distinct categories ( 8 images from each), such that two of the categories (i.e. body parts and clothing) were closer to each other than a third distant category (i.e. vehicles). For detailed analysis of the semantic distances between and within categories, see Vigliocco et al. (2002).

The structure of each session was also identical to Vigliocco et al. (2002). The study included 12 blocks repeated two times in pseudorandom order (each block sampled once before all blocks were repeated). Each block included 32 items: 8 items 
presented 4 times each in pseudorandom order (i.e. each item sampled once before items were repeated). A total of 768 stimuli were presented. Participants could take a break between each block. For the same condition, two different blocks were created for each of the three categories (total of 6 blocks), including items only from that category. Blocks that had images from the vehicles category were treated as fillers, while half the items (chosen randomly) were treated as fillers in blocks from the other two categories. For the near condition, two blocks were created with four randomly selected items from each of the near categories (i.e. body parts and clothing), such that individual items occurred equally often in all block types. Finally, for the far condition, two blocks were created for each combination: vehicles and body parts, and vehicles and clothing.

\section{Procedure}

This study was part of a project investigating the pharmacokinetics and tolerability of LSD and participants received between $40 \mathrm{mcg}$ and $80 \mathrm{mcg}$ of LSD intravenously. The protocol on both placebo and drug days was identical, except for a prolonged initial monitoring period on the drug day (45 mins vs. 20 mins, post-injection). Participants performed other tasks during the day and at least one week separated the placebo day from drug day, with placebo day occurring first in a single-blind manner. It must be noted that while the participants were blind to which day they received the drug, the effects of the drug are not subtle and this can theoretically break the blind. This sparks a recurrent debate regarding using an active placebo in experiments with LSD and similar drugs, but is beyond the scope of this paper (see Gouzoulis-Mayfrank et al., 1998a).

Participants performed the naming task on each experiment day. The task occurred in the same position relative to other tasks in the protocol, though exact task onset post-injection was not controlled (task onset: 180-300 minutes post-injection). Participants were asked to rate their subjective drug effects on a scale of 0 ('no effects') 
to 10 ('extremely intense effects') approximately every 30-45 minutes. At the beginning of the picture-naming task, participants reported drug effects that were on average less than one point less than the maximum reported effects. Drug effects had sufficiently diminished for the psychiatric release assessment at 5-6 hours post-dose.

The experimenter explained the task and mentioned that reaction times and accuracy would be assessed. The participant was asked to name each picture as quickly and as accurately as possible. In the practice session, each picture appeared randomly one by one for the participant to name. The experimenter pushed a key on an external keyboard to trigger the next image, and provided the name for the image if the participant failed to recognize a picture.

The participant continued on to the experimental blocks. Within each block, the experimenter triggered each trial to begin via an external keyboard as soon as the participant named an image from the previous trial. After each break, the participant told the experimenter to press the key to begin the next block.

In each 32-trial block, a fixation cross appeared at the center of the screen for $300 \mathrm{~ms}$, then a blank screen for $450 \mathrm{~ms}$, and the target image for $4000 \mathrm{~ms}$ or until the participant began producing a word. Once the participant began producing a word, the experimenter pressed the key for the next trial. At the end of each trial, the image disappeared and a blank screen appeared for $200 \mathrm{~ms}$ before the beginning of the next trial.

Stimuli were presented and each response was recorded as a separate .wav sound file using PsychoPy 1.78.01 (Pierce, 2007) on a PC laptop. Accuracy and error types were coded manually for all participants. The sound files were processed for response latencies via an automatic voice-onset detection script for Praat (Boersma, 2001). The 
automated response latencies were checked for accuracy against manually determined speech onset for all target items in both drug conditions for two participants.

\section{Results}

\section{Naming latencies}

We excluded filler blocks and the first instance of each item per block (for which semantic context effects should not yet occur) from the analysis of naming latencies. We also excluded all errors (243 trials) and sound files with sound disturbances (i.e. any sound other than speech that might affect the accuracy of speech onset detection, which were mainly due to malfunction of the laptop sound card used during the experiment, 569 trials), and trials where naming latencies were lower than $250 \mathrm{~ms}$ or higher than $2000 \mathrm{~ms}$ (37 trials). Mean reaction times were calculated for each semantic category for each session.

We then conducted a $2 \times 3$ ANOVA (drug condition $\times$ block type), separately for subjects $\left(\mathrm{F}_{1}\right)$ and items $\left(\mathrm{F}_{2}\right)$ as random effects. The main effect of drug condition was reliable only by items $\left(F_{1}(1,9)=1.77, p=.217, \eta_{p}{ }^{2}=.164 ; F_{2}(1,15)=16.47, p<.001, \eta_{p}{ }^{2}=\right.$ .523). The main effect of block type was significant $\left(\mathrm{F}_{1}(2,18)=5.76, \mathrm{p}=.012, \eta_{\mathrm{p}}{ }^{2}=.390\right.$, $\left.\mathrm{F}_{2}(2,30)=6.20, \mathrm{p}=.006, \eta_{\mathrm{p}}{ }^{2}=.293\right)$. The interaction was not significant $\left(\mathrm{F}_{1}(2,18)=2.86\right.$, $\left.\mathrm{p}=.083, \eta_{\mathrm{p}}{ }^{2}=.241 ; \mathrm{F}_{2}(2,30)=1.911, \mathrm{p}=.166, \eta_{\mathrm{p}}{ }^{2}=.113\right)$. Reaction times per drug per condition are plotted in Figure 2. We followed up the main effect of block type with planned pairwise comparisons. Naming latencies for the far condition were faster than the near condition $\left(\mathrm{t}_{1}(9)=2.79, \mathrm{p}=.021, \mathrm{t}_{2}(15)=3.08, \mathrm{p}=.008\right)$ and the same condition $\left(t_{1}(9)=2.84, p=.019, t_{2}(15)=3.88, p=.11\right)$. However, there were no differences between the near and same conditions (both $|\mathrm{t}|<1$ ). (Figure 2 about here) 


\section{Errors}

We classified errors into the following types: filled pauses before producing the correct word (e.g. "uhhhh leg” for "leg"), hesitations (stutter or dysfluency on otherwise correct production, e.g. "tru - truck" for "truck"), self-corrections (e.g. "trou .. uh.. shirt" for "shirt") and full lexical substitutions (e.g. "foot" for "leg"). The category of lexical substitutions also included cases where the subject uttered the correct word after the error was made (e.g., "shirt trousers" for "trousers"). We further classified lexical substitutions into same-category (e.g. "foot" for "leg") and different-category (e.g. "glove" for "hand"), as the latter are likely to arise due to visual similarity among pictures rather than during lexical retrieval. These results are summarized in Figure 3.

The total number of errors was low due to participants' high overall accuracy. We included all trials (fillers and targets) and as the distribution of errors was far from normally distributed, we used Wilcoxon signed rank tests, by subjects $\left(Z_{l}\right)$ and items $\left(Z_{2}\right)$ to test for effects of LSD. (Figure 3 about here)

Same-category lexical substitutions were significantly higher in the drug condition relative to the placebo condition $\left(Z_{1}=2.082, p=.038, Z_{2}=2.253, p=.006\right.$ (all $p$ values are two-tailed)). The effect of drug condition was not significant for selfcorrections, filled pauses, hesitations or lexical substitutions across category boundaries (all $|Z|<1)$.

We followed up with further tests involving same-category lexical substitutions. This effect appeared to be consistent across blocks $(|Z|<1$ for comparisons among blocks and interactions involving drug) so we continued to combine data across blocks. For the analysis of block type, we had to convert the number of errors into proportions (as filler items appeared mostly in the same condition and never in the near condition), presented in Table 1. (Table 1 about here) 
The main effect of condition on same-category lexical substitutions was investigated using pairwise Wilcoxon signed rank tests on proportion of errors. These errors did not differ for far and near conditions $(|Z|<1)$, but approached significance when individual conditions were compared with same (same - far: $Z_{1}=1.894, p=.058$; $Z_{2}=2.404, p=.016$; same-near: $\left.Z_{I}=1.958, p=.050, Z_{2}=1.965, p=.049\right)$. Far and near conditions were combined to test for an interaction between block type and drug, using Wilcoxon signed rank tests comparing the difference scores (LSD same - LSD not same) vs (Placebo same - Placebo not same). This interaction was significant only by subjects and not items $\left(Z_{1}=2.191, p=.028 ; Z_{2}=1.363, p=.173\right)$ suggesting that the appearance of an interaction between drug condition and block type may be present only in a subset of items rather than being general in nature.

\section{Discussion}

This study investigated the effects of the serotonergic hallucinogen LSD on lexical retrieval in a naming task. A previous study using the related drug psilocybin, found an increase in indirect semantic priming in the drug condition (Spitzer et al., 1996) suggesting an expanded semantic spread. We hypothesized that LSD would reveal similar effects in a picture naming task. Lower reaction times for far category blocks relative to those for same category blocks in both LSD and placebo conditions replicates findings reported by Vigliocco et al. (2002), demonstrating a clear context effect. While between-condition differences in reaction times were non-significant, LSD did have a significant effect on naming errors, and this effect was highly selective; there were significantly more substitution errors for semantically similar items under LSD (but not semantically different items) and this relationship was not modulated by block order or semantic condition. These error patterns suggest that LSD does in fact enhance the spread of semantic network activation, such that same-category items are more 
activated and therefore more prone to be produced as errors.

To our knowledge, this study is the first study of semantic effects to incorporate error measures in a pharmacological challenge, and can thus provide new insight and perspective in this context. Errors may be more sensitive to changes in activation patterns than reaction time when examining drug-induced modulation of semantic activation patterns in production.

Error detection is reflective of self-monitoring and we suggest that LSD may reduce this function, such that full lexical errors are produced that are not rapidly selfcorrected. Reduced error-correction under LSD is broadly consistent with previous findings of impaired attention under psilocybin (Carter et al. 2005). Moreover, some of the participants' comments in the present study are suggestive of an inability to focus attention under LSD: "sometimes I'll feel a bit slow because my brain has been off somewhere else and I have to bring it back to focus."; "the perception of the body is somewhat magnified and this can create challenges in focus or attention;" and: "I was actually having a little experiment of how much I can think of other things while doing the task." These comments may imply a particularly general effect of LSD on cognition, stimulating flexible and associative thinking while compromising the ability to attend and focus. However, looking more carefully at our error patterns, we note that while full substitution errors were more frequent in the LSD condition, errors reflecting early detection of mistakes (i.e. hesitations and self-corrections) were no different in the drug and placebo conditions (see Figure 3 above). These findings suggest that a selfmonitoring account of the error patterns is not sufficient, as we would expect fewer errors of these kinds in the drug condition as they arise from detection of errors or possible errors via self-monitoring (at least in some cases of hesitations, and certainly 
for self-corrections). Furthermore, reaction times are similar and not significantly different between drug and control groups.

The strength of behavioural paradigms in psychology research is that they force scientists to be clear about their definitions of phenomena and the questions they intend to ask, and they also encourage the use of well thought-through, controlled experiments that can test phenomena in an objective way. However, it is worth considering the limitations of this approach when studying a compound whose principal action seems to be on spontaneous as opposed to evoked processes (Muthukumuraswarmy et al., 2013). The incorporation of measures that sample spontaneous language production under psychedelics may be a more natural, practical, and informative approach therefore. Perhaps more diverse pictures with less repetition could be used in order to allow for more semantically diverse errors. Or participants could be encouraged to speak freely about a particular picture that is shown to them, as is done in the Thematic Apperception Test (TAT) for example (Bellak \& Abrams, 1997). This approach may produce less structured yet more natural language production that could reveal widerranging semantic activation at the sentence or discourse level. Future studies may benefit from utilizing complementary methods, incorporating both controlled and naturalistic measures of linguistic/sematic processing in order to better understand the effects of LSD on cognition. Furthermore, neuroscientific methods, such as the N400 event-related potential (Kutas \& Federmeier, 2011) would enable us to test the semantic spread of activation more formally and objectively (Holcomb, 1998). Nevertheless, even in the highly constrained experimental situation in which a small set of pictures were repeatedly named, effects consistent with increased spread of semantic activation due to LSD were observed, in line with Spitzer et al. (1996) for psilocybin. 
Some more specific limitations of the present study include: the small sample size, variable dosing and variations in the time that the task was performed postadministration. Explaining the first two factors, this was a pilot study, the main intention of which was to determine an appropriate dose of LSD for a subsequent neuroimaging study. Variations in task-onset were unfortunate but largely determined by participants differing in the time they took to complete previous tasks and their desire to talk to the experimenters. Spitzer et al. (1996) administered their picture naming task four times in the course of two hours and forty minutes post-ingestion of psilocybin and found a significant indirect semantic priming effect at the 50 minute testing point, arguing that this coincides with peak of plasma levels of the active drug. In the current study, the beginning of the naming task was more variable on the LSD day and we did not analyse plasma concentrations of LSD. However, depreciating the importance of the above-listed potential cofounds, neither time of task performance nor dosage correlated with magnitude of the main study outcomes.

\section{Conclusions}

In line with previous findings on the effects of psychedelics, between-condition differences in error and correction rates in the present study suggest that LSD and related psychedelics increase the spread of semantic activation. Further work is required to test the reliability and specificity of this effect, especially in light of the interplay between self-monitoring and the types of errors produced by the participants. This may be achieved by using more naturalistic approaches or looking at changes in electrophysiology. However, the current findings are broadly consistent with the notion that psychedelics alter the breadth and flexibility of cognition (Carhart-Harris et al., 2014a; Petri et al., 2014). 


\section{Acknowledgements}

This work was supported by the Deutsche Forschungsgemeinschaft under Grant FA 1234/1-1.

We would like to thank Alexis Hervais-Adelman for providing the automatic speech onset detection code for Praat that was used in the analysis.

\section{References}

Aarsland, D., Larsen, J., Reinvang, I., \& Aasland, A. (1994). Effects of cholinergic blockade on language in healthy young women. Brain, 117, 1377-1384. doi: 10.1093/brain/117.6.1377

Aghajanian, G.K., \& Bing, O.H.L. (1964). Persistence of lysergic acid diethylamide in the plasma of human subjects. Clinical Pharmacology and Therapeutics, 5, 611614. doi:10.1002/cpt196455611

Albert, M. L. (2000). Toward a neurochemistry of naming and anomia. In Y. Grodzinsky, L. Shapiro, \& D. Swinney (Eds.), Language and the Brain: Representation and Processing (157-165). San Diego, CA: Academic Press.

Amarel M., \& Cheek F.E. (1965). Some effects of LSD-25 on verbal communication. Journal of Abnormal Psychology, 70 (6), 453-456.

Bellak, L., \& Abrams, D. M. (1997). The Thematic Apperception Test, the Children's Apperception Test, and the Senior Apperception Technique in Clinical Use. Boston, MA: Allyn \& Bacon.

Boersma, P. (2001). Praat, a system for doing phonetics by computer. Glot International $5: 9 / 10,341-345$.

Carhart-Harris, R.L., Leech, R., Hellyer, P.J., Shanahan, M., Feilding, A., Tagliazucchi, E., Chialvo, D.R., Nutt, D.J. (2014a). The entropic brain: A theory of conscious states informed by neuroimaging research with psychedelic drugs. Frontiers in Human Neuroscience, 8:20. doi:10.3389/fnhum.2014.00020

Carhart-Harris, R.L., Kaelen, M., \& Nutt, D. (2014b). How do hallucinogens work on the brain? The Psychologist, 27(9), 662-665.

Carter, O.L., Burr D.C., Pettigrew, J.D., Wallis, G.M., Hasler, F., \& Vollenweider, F.X. (2005). Using psilocybin to investigate the relationship between attention, working memory, and the serotonin 1A and 2A Receptors. Journal of Cognitive Neuroscience, 17(10), 1497-1508. doi:10.1162/089892905774597191 
Copland, D. A., Chenery, H. J., Murdoch, B. E., Arnott, W. L., \& Silburn, P. A. (2003). Dopamine enhances semantic salience: Semantic priming evidence from healthy individuals. Brain and Language, 87, 103-104. doi:10.1016/S0093934X(03)00220-7

Damian, M. F., Vigliocco, G., \& Levelt, W. J. M. (2001). Effects of semantic context in the naming of pictures and words. Cognition, 81, B77-B86. doi:10.1016/S00100277(01)00135-4

Dhooge, E., Baene, W. D., \& Hartsuiker, R. J. (2013). A late locus of the distractor frequency effect in picture-word interference: Evidence from event-related potentials. Brain and Language, 124, 232-237. doi:10.1016/j.band1.2012.12.005

Fromkin, V. A. (1971). The non-anomalous nature of anomalous utterances. Language, 47(1), 27-52. doi:10.2307/412187

Garrett, M. F. (1992). Lexical retrieval processes: Semantic field effects. In A. Lehrer, E.F. Kittay \& R. Lehrer (Eds.), Frames, fields, and contrasts: New essays in semantic and lexical organization, (377-395). Hillsdale, NJ: Lawrence Erlbaum Associates, Inc.

Gasser, P., Holstein, D., Michel, Y., Doblin, R., Yazar-Klosinski, B., Passie, T., \& Brenneisen, R. (2014). Safety and efficacy of lysergic acid diethylamide-assisted psychotherapy for anxiety associated with life-threatening diseases. Journal of Nervous and Mental Disease, 202(7), 513-520.

doi:10.1097/NMD.0000000000000113.

Geyer M.A., \& Vollenweider F.X. (2008). Serotonin research: contributions to understanding psychoses. Trends in Pharmacological Sciences, 29(9), 445-53. doi: $10.1016 /$ j.tips.2008.06.006

Gouzoulis-Mayfrank, E., Schneider, F., Spitzer, M., Thelen, B., \& Sass, H. (1998a). Methodological issues of human experimental research with hallucinogens. Pharmacopsychiatry, 31(S2), 114-118. doi:10.1055/s-2007-979356

Gouzoulis-Mayfrank, E., Thelen, B., Maier, S., Habermeyer, E., Spitzer, M. \& Sass, H. (1998b). Modulation of semantic priming effects by psilocybin, MDE (ecstasy), and d-methamphetamine: Principles and applications of the model psychosis strategy. Neurology Psychiatry and Brain Research, 6, 19-28. doi:10.1159/000063672 
Halberstadt, A.L., \& Geyer, M.A. (2011). Multiple receptors contribute to the behavioral effects of indoleamine hallucinogens. Neuropharmacology, 61(3), 364-81. doi: 10.1016/j.neuropharm.2011.01.017

Hantsch, A., \& Mädebach, A. (2013). What does the articulatory output buffer know about alternative picture names? Evidence against the response-exclusion hypothesis. Language and Cognitive Processes, 28, 684-700. doi:10.1080/01690965.2011.595725

Harvey, J. A. (2003). Role of the serotonin 5- $\mathrm{HT}_{2 \mathrm{~A}}$ receptor in learning. Learning and Memory, 10, 355-362. doi:10.1101/1m.60803

Hasler, F., Grimberg, U., Benz, M.A., Huber, T., \& Vollenweider, F.X. (2004). Acute psychological and physiological effects of psilocybin in healthy humans: a double-blind, placebo-controlled dose-effect study. Psychopharmacology, 172, 145-56. doi:10.1007/s00213-003-1640-6

Holcomb, P.J. (1998). Automatic and attentional processing: An event-related brain potential analysis of semantic priming. Brain and Language, 35, 66-85. doi:10.1016/0093-934X(88)90101-0

Landon, M., \& Fischer, R. (1970). On similar linguistic structures in creative performance and psilocybin-induced experience. Confinia Psychiatrica, 13, 115138.

Kischka, U., Kammer, T., Maier, S., Weisbrod, M., Thimm, M., \& Spitzer, M. (1996). Dopaminergic modulation of semantic network activation. Neuropsychologia. 34(11), 1107-1113. doi:10.1016/0028-3932(96)00024-3

Kometer, M., Cahn, B.R., Andel, D., Carter, O.L., \& Vollenweider, F.X. (2011). The 5HT2A/1A agonist psilocybin disrupts modal object completion associated with visual hallucinations. Biological Psychiatry, 69, 399-406. doi:

10.1016/j.biopsych.2010.10.002

Kroll, J. F., \& Stewart, E. (1994). Category interference in translation and picture naming: evidence for asymmetric connections between bilingual memory representations. Journal of Memory and Language, 33, 149-174. doi:10.1006/jmla.1994.1008

Kutas, M., \& Federmeier, K. D. (2011). Thirty years and counting: Finding meaning in the N400 component of the event related brain potential (ERP). Annual Review of Psychology, 62, 621. doi:10.1146/annurev.psych.093008.131123 
Mahon, B. Z., Costa, A., Peterson, R., Vargas, K. A., \& Caramazza, A. (2007). Lexical selection is not by competition: a reinterpretation of semantic interference and facilitation effects in the picture-word interference paradigm. Journal of Experimental Psychology: Learning, Memory, and Cognition, 33, 503-535. doi: 10.1037/0278-7393.33.3.503

Muthukumuraswarmy, S., Carhart-Harris, R.L., Moran, R.J., Brookes, M.J., Williams, T.M., Errtizoe, D., Sessa, B., Papadopoulos, A., Bolstridge, M., Singh, K.D., Feilding, A., Friston, K.J., Nutt, D.J. (2013). Broadband cortical desynchronization underlies the human psychedelic state. Journal of Neuroscience, 33(38), 15171-15183. doi: 10.1523/JNEUROSCI.2063-13.2013

Navarrete, E., Mahon, B. Z., \& Caramazza, A. (2010). The cumulative semantic cost does not reflect lexical selection by competition. Acta Psychologica, 134(3), 279-289. doi:10.1016/j.actpsy.2010.02.009

Peirce, J.W. (2007). PsychoPy - Psychophysics software in Python. Journal of Neuroscience Methods, 162(1-2), 8-13. doi:10.1016/j.jneumeth.2006.11.017

Petri, G., Expert, P., Turkheimer, F., Carhart-Harris, R., Nutt, D., Hellyer, P. J., \& Vaccarino, F. (2014). Homological scaffolds of brain functional networks. Journal of The Royal Society Interface, 11(101). doi: 10.1098/rsif.2014.0873

Raote, I., Bhattacharya, A., \& Panicker, M.M. (2007). Serotonin 2A (5-HT2A) Receptor Function: Ligand-Dependent Mechanisms and Pathways. In A. Chattopadhyay (Ed.), Serotonin Receptors in Neurobiology (Chapter 6). Boca Raton, FL: CRC Press.

Rosenberger, P. (1980). Dopaminergic systems and speech fluency. Journal of Fluency Disorders, 5, 255-267. doi:10.1007/s00702-015-1363-y

Schriefers, H., Meyer, A. S., \& Levelt, W. J. M. (1990). Exploring the time course of lexical access in language production: picture-word interference studies. Journal of Memory and Language, 29, 86-102. doi:10.1016/0749-596X(90)90011-N

Snodgrass, J. G., \& Vanderwart, M. (1980). A standardized set of 260 pictures: norms for name agreement, image agreement, familiarity, and visual complexity. Journal of Experimental Psychology: Learning, Memory and Cognition, 6, 174215. doi: 10.1037/0278-7393.6.2.174

Spitzer, M., Thimm, M., Hermle, L., Holzmann, P., Kovar, K. A., Heimann, H., Gouzoulis-Mayfrank, E., Kischka, U., Schneider F. (1996). Increased activation 
of indirect semantic associations under psilocybin. Biological Psychiatry, 39, 1055-1057. doi:10.1016/0006-3223(95)00418-1

Sundheim, S.T., \& Voeller, K.K. (2004). Psychiatric implications of language disorders and learning disabilities: risks and management. Journal of Child Neurology, 19(10), 814-826. doi: 10.1177/08830738040190101001

Vigliocco, G., Vinson, D. P., Damian, M. F., \& Levelt, W. (2002). Semantic distance effects on object and action naming. Cognition, 85, B61-B69. doi:10.1016/S0010-0277(02)00107-5

Williams, G. V., Rao S. G., \& Goldman-Rakic, P. S. (2002). The physiological role of 5-HT2A receptors in working memory. Journal of Neuroscience, 22, 2843-2854.

Wolbach, A. B., Miner E.J., \& Isbell, H. (1962). Comparison of Psilocin with Psilocybin, Mescaline and LSD-25. Psychopharmacologia, 3, 219-223. doi:10.1007/BF00412109 
Table 1. Number of same-category lexical substitutions as a function of drug condition and block type (percentage in brackets).

\begin{tabular}{|c|c|c|}
\hline & LSD & PLACEBO \\
\hline Far & $27(1.1 \%)$ & $21(0.8 \%)$ \\
\hline Near & $11(0.8 \%)$ & $10(0.7 \%)$ \\
\hline $\mathrm{am}$ & $68(1.8 \%)$ & $36(0.9 \%)$ \\
\hline
\end{tabular}


Figure 1. Indirect priming and plasma psilocin level relative to time post-ingestion in Spitzer et al. (1996)

Figure 2. Trimmed correct naming latencies as a function of block type $\times$ drug condition. Error bars reflect standard error of the estimated cell mean, calculated by subject ( $F_{1}$ analysis)

Figure 3. Percentage of different types of errors as a function of drug condition, * significant difference $(\mathrm{p}<.01)$ 


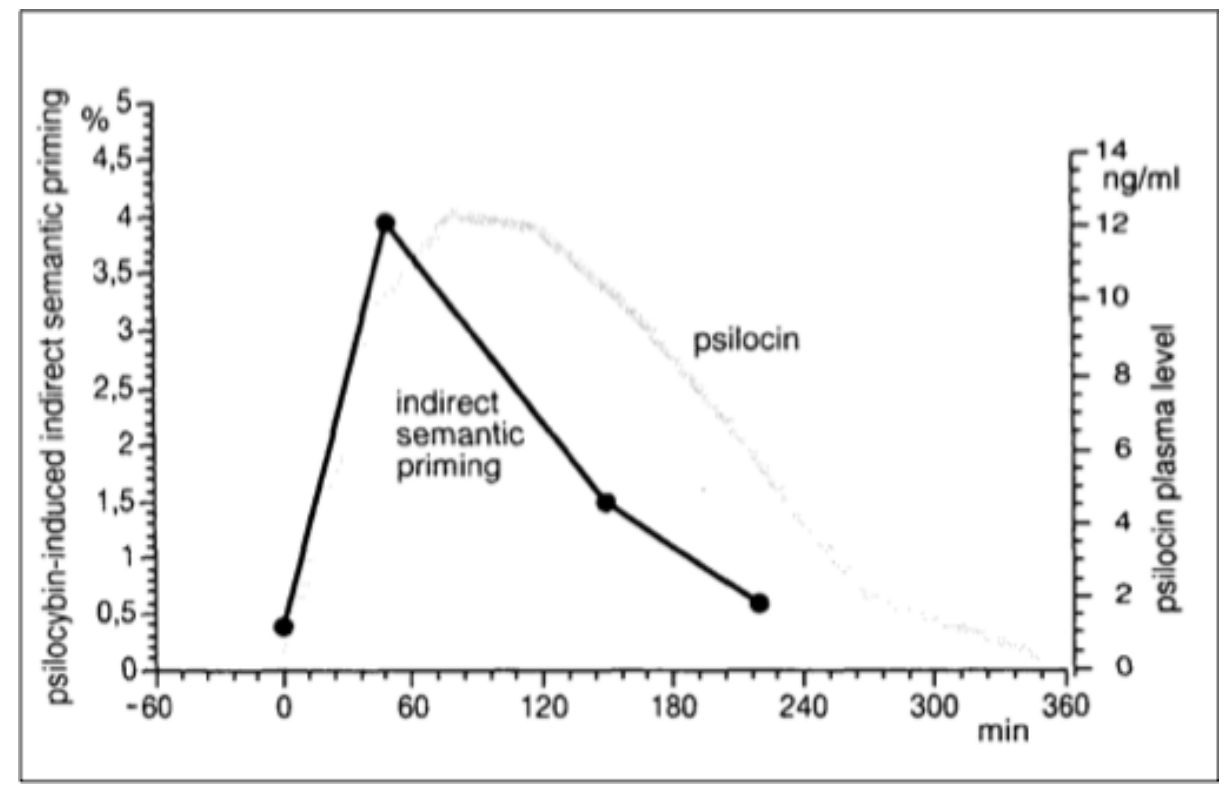




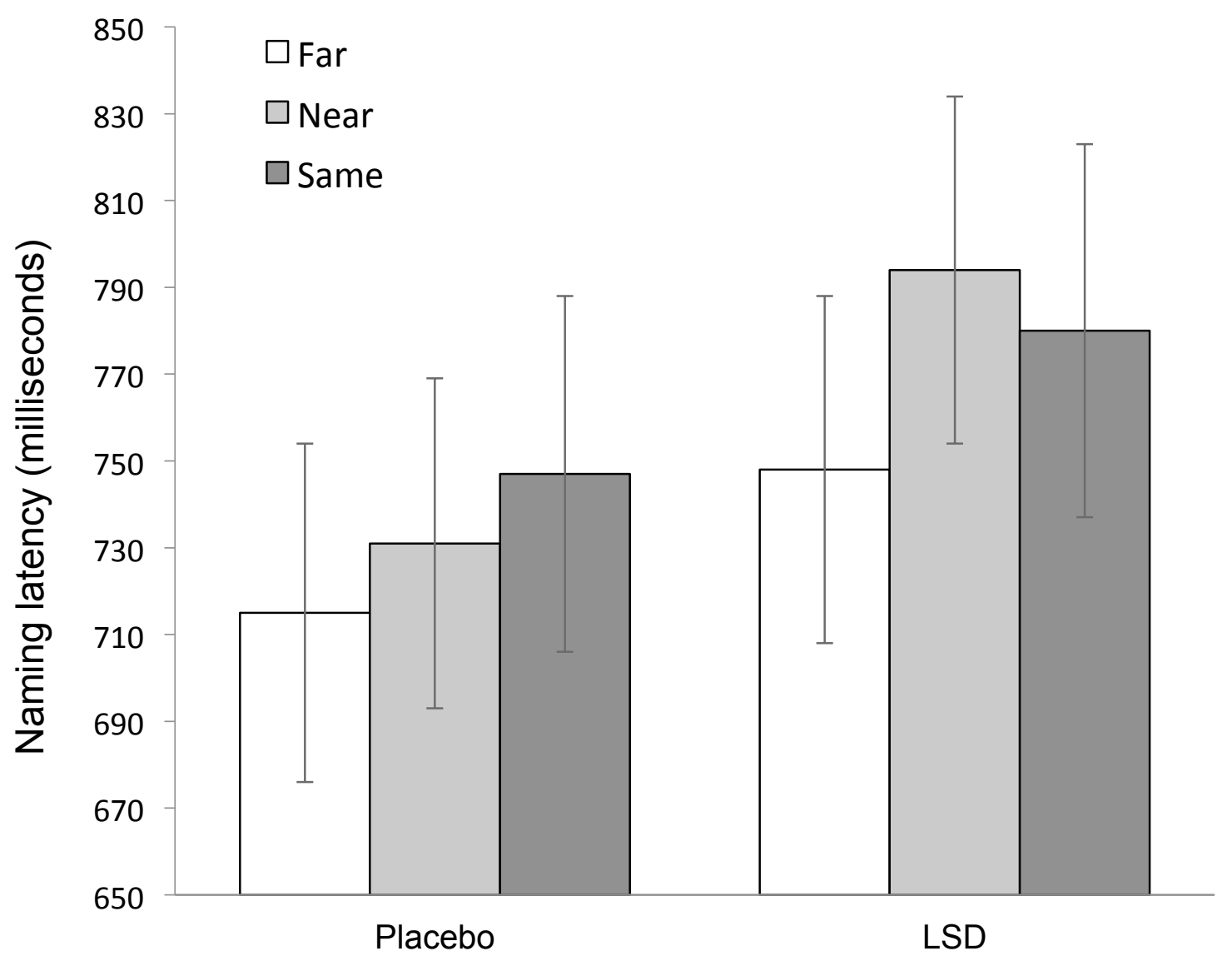




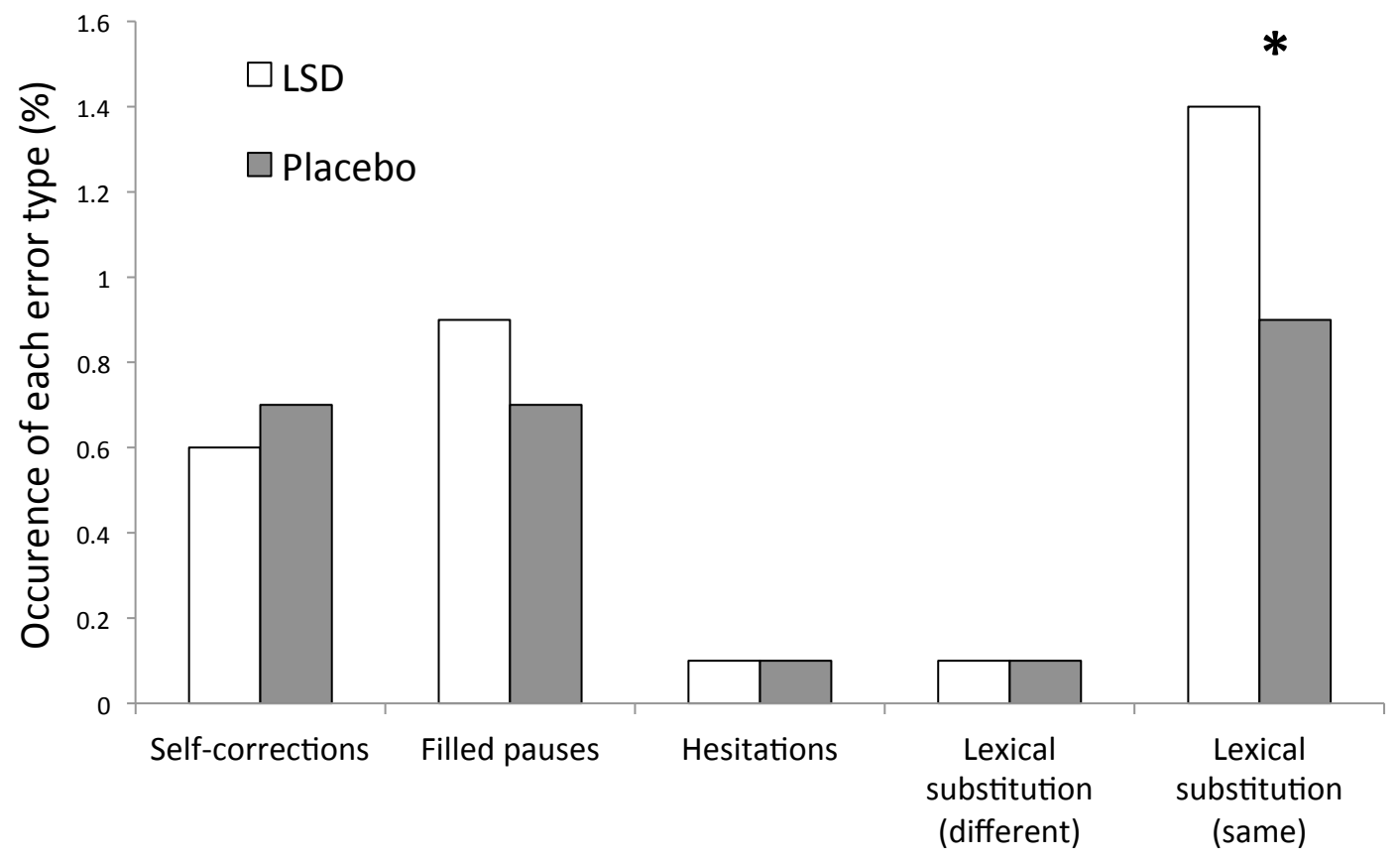

